\title{
Manejo multidisciplinar del cáncer epitelial de ovario. Diagnóstico radiológico
}

\section{Multidisciplinary management of ovarian epithelial cancer. Radiological diagnosis}

\author{
M.C. Sánchez, J. Sáenz, S. Ostiz
}

\section{RESUMEN}

En el contexto del abordaje multidisciplinar para el diagnóstico y tratamiento del cáncer de ovario, la aportación del radiólogo incluye cuatro puntos fundamentales que son: identificación de la lesión, caracterización, estudio de extensión y valoración evolutiva. En el presente trabajo se describen los hallazgos y claves diagnósticas en TCMD (Tomografía Computarizada multidetector) y RM (Resonancia Magnética), la aportación e indicación de otras tecnologías como el PET (Tomografía por Emisión de Positrones) y se esbozan las técnicas emergentes basadas en imagen funcional (estudios dinámicos con contraste en RM y estudios de difusión por RM).

Palabras clave. Cáncer epitelial de ovario. Resonancia magnética. Tomografía computarizada multidetector.

\begin{abstract}
In the context of a multidisciplinary approach for the diagnosis and treatment of ovarian cancer, the contribution of radiology includes the following four fundamental points: identification of the lesion, its characterization, study of its extension and evaluation of its evolution. This article describes the findings and key diagnostic elements in MDCT (Multidetector Computed Tomography) and MR (Magnetic Resonance), the contribution and indication of other technologies like PET (Positron Emission Tomography), and outlines the emergent techniques based on functional image analysis (dynamic contrast-enhanced MRI studies and MRI diffusion studies).
\end{abstract}

Key words. Ovarian Epithelial Cancer. Magnetic Resonance. Multidetector Computed Tomography.
Servicio de Radiología B.

Complejo Hospitalario de Navarra

Recepción: 2 de febrero de 2011

Aceptación provisional: 5 de mayo de 2011

Aceptación definitiva: 17 de mayo de 2011

\section{Correspondencia}

María Carmen Sánchez Rodríguez

Complejo Hospitalario de Navarra

Servicio de Radiología B

Irunlarrea, 4

31008 Pamplona

E-mail: csancher@cfnavarra.es 


\section{IDENTIFICACIÓN DEL TUMOR OVÁRICO}

La clave para la identificación de las lesiones se basa en el reconocimiento de la anatomía y la reproducción que de la misma hacen las técnicas de imagen, fundamentalmente la TC y RM. El objetivo es identificar el ovario y definir la dependencia ovárica o no de la masa o lesión en estudio. Reconoceremos el ovario por su morfología y localización siguiendo el curso de los vasos ováricos y el anclaje de sus ligamentos.

El ovario es un órgano par de morfología ovalada que contiene folículos y cuyo tamaño varía en función de la edad reproductiva de la paciente. Los diámetros aproximados en la mujer adulta son 2,5-5 $\mathrm{cm}$ longitud $\mathrm{x}$ 1,5-3 $\mathrm{cm}$ anchura y $1-2 \mathrm{~cm}$ de grosor, prefiriéndose en la actualidad la valoración del volumen calculado con la fórmula (0,53 x long $\mathrm{x}$ ancho $\mathrm{x}$ grosor). El volumen promedio del ovario obtenido por US a partir de series amplias es de $3,0 \mathrm{ml}$ antes de la menarquia, $9,8 \mathrm{ml}$ en edad fértil y $5,8 \mathrm{ml}$ en mujeres postmenopáusicas.

Su localización es pélvica intraperitoneal y está anclado por una serie de ligamentos:

- El mesoovario que lo sujeta a la cara posterior del ligamento ancho, ocasionalmente visible en TC cuando hay ascitis, que a su vez divide la pelvis profunda en dos compartimentos, anterior y posterior.

- El ligamento uteroovárico que lo une a la pared lateral del útero.

- El ligamento suspensorio o infundibulopélvico, formado por un repliegue peritoneal desde la parte superolateral del ligamento ancho con morfologia triangular o de abanico, entrecruzado con tejido fibromuscular, que ancla el ovario a la pared posterolateral de la pelvis (el que mejor se identifica mediante TC).

Estos ligamentos tienen laxitudes variables lo que condiciona una posición diferente del ovario en distintas pacientes e incluso en una misma paciente en momentos distin- tos. Esta característica supone un reto para el radiólogo a la hora de identificar el ovario en el TC, siendo la clave el seguimiento de los vasos ováricos y fundamentalmente de la vena ovárica, ya que la arteria aunque sigue un trayecto paralelo tiene un diámetro menor. La vena ovárica izquierda drena en la vena renal ipsilateral y es algo mayor de calibre que la contralateral, que desemboca directamente en la vena cava. Las arterias ováricas emergen de la aorta por debajo de las renales. Los vasos ováricos discurren por delante del músculo psoas, cruzando de medial a lateral y en descenso por delante del uréter hasta alcanzar la porción lumbar baja y pélvica, atravesando posteriormente el ligamento infundibulopélvico para contactar con el ovario a través del mesoovario (Fig. 1).

El hecho de que el mesoovario se encuentre anclado a la pared posterior del ligamento ancho obliga a buscar el ovario en el compartimento pélvico posterior, hallándose lateral, superior o posterior al útero, pero nunca entre útero y vejiga.

Otro criterio para discernir la dependencia de una lesión, es su relación con las estructuras de vecindad, de esta forma una masa de origen ovárico se localiza anterior y medial al uréter y si es grande produce un desplazamiento del mismo en sentido lateral o posterolateral, a diferencia de lo que ocurre con los conglomerados de adenopatías iliacas que pueden englobar los vasos iliacos y desplazan el uréter en sentido anteromedial. Si existe suficiente cantidad de grasa pélvica podremos identificar el peritoneo parietal posterior y por detrás de éste los vasos y los ganglios. Este dato permite establecer diferencias entre las masas ováricas y las adenopatías, pero un útero aumentado de tamaño o incluso la vejiga o masas dependientes de intestino pueden desplazar el uréter en el mismo sentido, teniendo mayor fiabilidad para determinar la dependencia ovárica de una lesión la comprobación de que los vasos ováricos penetran en la misma. Los falsos positivos se producen con masas de gran tamaño que comprimen los vasos ováricos y parece que penetran en la masa. En este 
caso conseguir localizar el ovario normal fuera de la lesión, asegura el diagnóstico de masa extraovárica. Por otra parte dada la íntima relación entre el ovario y la trompa no es posible diferenciar dependencias de una u otra estructura ${ }^{1}$.

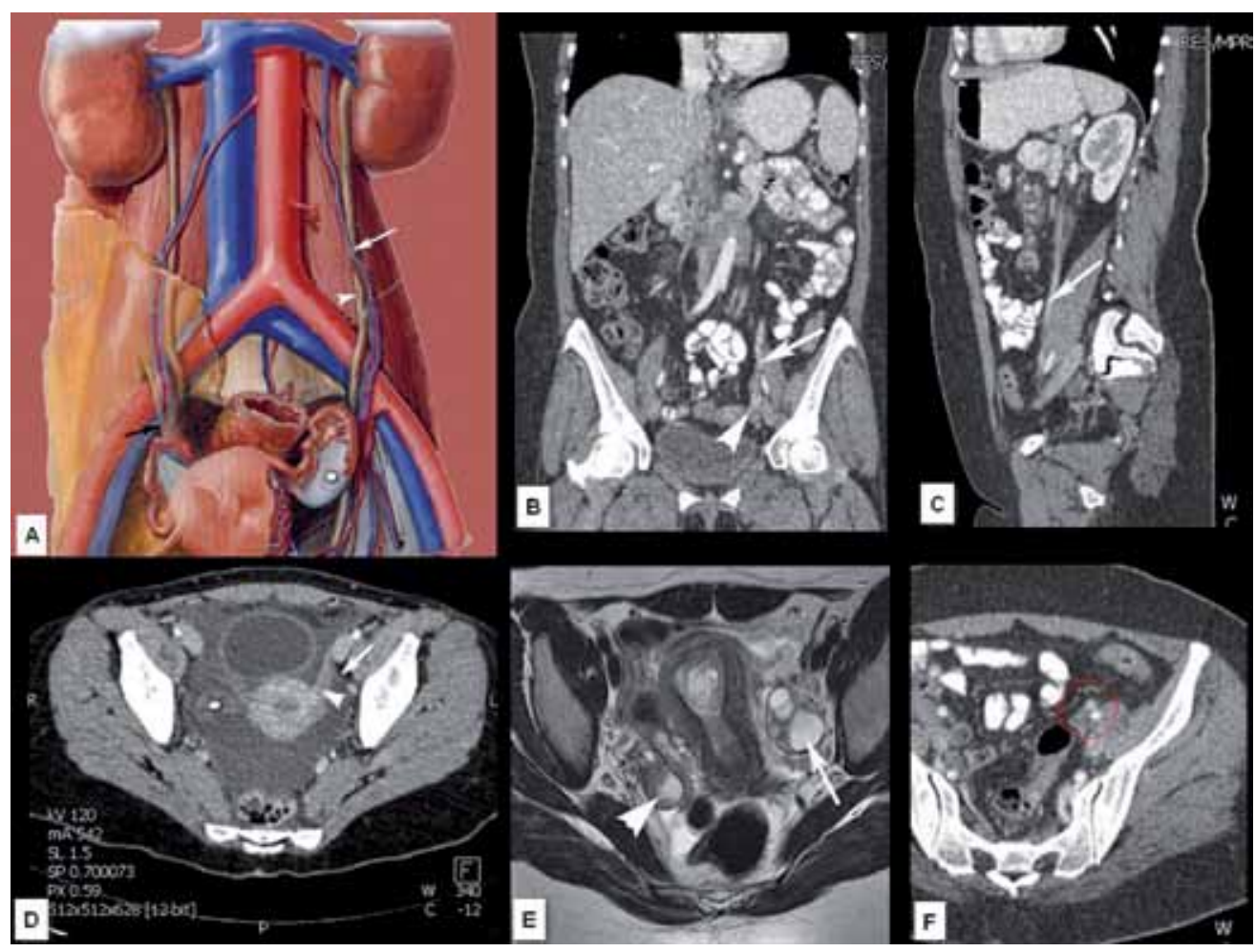

Figura 1. Correlación anatomo-radiológica. A: Esquema anatómico en el que se señalan los vasos ováricos (flecha blanca), el uréter (cabeza de flecha), el ligamento infundibulopélvico (flecha negra) y el ovario (punto blanco). B: TC coronal. Vena ovárica izquierda (flecha) y ligamento infundibulopélvico (cabeza de flecha). C: TC sagital. Vena ovárica (flecha). D: TC axial. Ascitis que permite identificar el ligamento ancho (cabeza de flecha), peritoneo parietal posterior (flecha) y el ovario (punto blanco). E: RM axial. Se identifican ambos ovarios con múltiples folículos (flecha y cabeza de flecha). F: TC axial. Ligamento infundibulopélvico con su morfología triangular habitual (círculo rojo).

\section{CARACTERIZACIÓN DEL TUMOR OVÁRICO}

El cáncer del ovario sigue siendo la primera causa de muerte dentro de las neoplasias ginecológicas. La mayoría de las pacientes son diagnosticadas en estadios avanzados (estadio III) con una supervivencia a los 5 años entre el $30-73 \%$. Mientras que las recientes diagnosticadas en es- tadio I tienen una supervivencia superior al $90 \%$ a los 5 años, manteniéndose por tanto, la necesidad de un diagnóstico precoz ${ }^{2}$.

Los múltiples intentos de cribaje sobre población asintomática se han demostrado ineficaces debido a la alta incidencia de masas ováricas benignas tanto en mujeres pre como postmenopáusicas ${ }^{2,3}$, lo que, junto con la baja incidencia de cáncer en la población general, hace que un test 
diagnóstico con una sensibilidad del 100\% y una especificidad del $99 \%$, tenga un valor predictivo positivo del $4,8 \%$. Luego más del 95\% de las lesiones detectadas serán benignas, o falsos positivos.

La técnica de primera elección para el estudio de las lesiones ováricas es la ecografia transvaginal con cifras del $85-100 \%$ de sensibilidad y un $52-100 \%$ de especificidad para el diagnóstico de cáncer. La gran variabilidad de la prueba está influida por el hábito corporal de las pacientes y por ser una prueba operador dependiente, lo que hace necesario un segundo test para la caracterización más precisa de las lesiones indeterminadas en ultrasonidos (US) ${ }^{2,4}$.

Por otra parte el cáncer más comúnmente encontrado en la práctica clínica es el cistoadenocarcinoma seroso de alto grado, que se origina en la superficie del ovario y es rápidamente progresivo desde su estadio precoz, detectable por ecografia, al avanzado (estadio III). Algunos estudios han demostrado crecimiento en 4-6 semanas con una estimación de tiempo de doblaje menor de 3 meses.

Dado el rápido avance y propensión a diseminarse del cáncer de ovario, la pauta de actuación consensuada es que si una masa no puede ser caracterizada como benigna o si hay indicadores o factores de riesgo que sugieren cáncer, la lesión será resecada en lugar de realizar seguimiento, no siendo asumible el riesgo de control evolutivo que se practica con lesiones indeterminadas en otras localizaciones del organismo. No obstante, debemos tener en cuenta la morbi-mortalidad asociada a la ooforectomía uni o bilateral (adherencias, hidrosalpinx, infecciones, reducción de la fertilidad, menopausia prematura con pérdida de masa ósea e incremento de riesgos cardiovasculares), siendo ésta la razón por la que resulta importante la caracterización de las lesiones con objeto de evitar cirugías innecesarias ${ }^{2}$.

La gran mayoría de los tumores del ovario entran dentro de 3 categorías:

- Tumores epiteliales de la superficie que suponen el $60 \%$ de los tumores del ovario y el $85-90 \%$ de los malignos.
- Tumores de los cordones sexuales.

- Tumores de células germinales.

Los tumores epiteliales nacen de la superficie epitelial y son clasificados anatomopatológicamente como serosos, mucinosos, células claras, endometrioides o tumor de Brenner (transicional), y cada uno de ellos en benignos, malignos, o borderline lo que se reflejará en diferentes comportamientos clínicos.

La caracterización de las masas ováricas por parte del radiólogo debe incluir el diagnóstico diferencial con las metástasis ováricas (10\% de todos los tumores del ovario $)^{5}$ siendo este aspecto de suma importancia por la implicación pronóstica y terapéutica (tratamiento quirúrgico incluso en pacientes con carcinomatosis de origen ovárico y no quirúrgica en las metástasis y carcinomatosis de origen digestivo $)^{2,6}$.

Aunque tanto las manifestaciones clínicas como radiológicas de estos tumores pueden solaparse, existen algunos hallazgos que aportan especificidad y permiten el diagnóstico diferencial dentro de distintos tipos histológicos. No obstante, la caracterización más importante y que continúa siendo un desafío para la imagen es la diferenciación entre benigno-maligno.

Los radiólogos disponemos de dos técnicas de imagen que son la TC y la RM mediante las que valoramos tanto masas incidentales del ovario como lesiones indeterminadas por ecografía. Mientras la TC es la técnica de elección en el estudio de extensión del cáncer de ovario y estimación de la posibilidad de cirugía óptima, la RM ha demostrado mayor eficacia en la caracterización de las masas ováricas, basado en su mayor capacidad de discriminación tisular".

Esto permite diferenciar con fiabilidad suficiente determinados tipos histológicos y proporciona información tanto de masas no neoplásicas como neoplásicas y en este caso su benignidad o malignidad.

Uno de los roles más importantes de la RM es distinguir entre benigno y maligno. Existe un metaanálisis que evalúa el valor añadido de un segundo test para masas 
anexiales indeterminadas vistas por eco transvaginal, y determina que la RM con contraste IV proporciona la mayor probabilidad post-test de cáncer de ovario cuando se compara con TC, eco Doppler, o RM sin contraste. En esta situación demuestra una sensibilidad del $100 \%$ y una especificidad del $94 \%$ en el diagnostico de malignidad ${ }^{2}$. La administración de Gd, aunque no se utiliza de forma rutinaria, ha mejorado la precisión diagnóstica en determinados casos con sospecha de transformación maligna de endometriomas (carcinoma de células claras, carcinoma endometrioide) o teratomas quísticos (carcinoma escamoso invasivo) ${ }^{6,7}$.

Aunque la RM puede ayudar en la detección, lo realmente importante es su especificidad porque proporciona un diagnóstico seguro de muchas lesiones anexiales benignas. La TC y la RM son iguales en sensibilidad pero, la RM presenta una especificidad del $98 \%$ frente al $87 \%$ de la TC. El valor predictivo positivo de la RM es del $44,18 \%$ frente al $6,81 \%$ de la $\mathrm{TC}^{8}$. En un estudio prospectivo de mujeres con sospecha de masas anexiales, tanto el Doppler como la RM demostraron una alta sensibilidad (US 100\% y RM 96,6\%), pero la especificidad de la RM fue notablemente mayor (US $39,5 \%$ RM 83,7\%). Por lo tanto, las mujeres que clínicamente tienen un bajo riesgo de malignidad pero tienen una lesión indeterminada son quienes más se benefician del estudio mediante $\mathrm{RM}^{2}$.

Tabla 1. Clasificación morfológica

\begin{tabular}{lll}
\hline & \multicolumn{1}{c}{ Benignas } & \multicolumn{1}{c}{ Malignas } \\
\hline \multirow{3}{*}{ Masas quísticas } & $\begin{array}{l}\text { Quiste funcionales } \\
\text { Quistes de inclusión } \\
\text { Quistes del paraovario } \\
\text { Quistes paratubáricos } \\
\text { Hidrosalpinx }\end{array}$ & Excepcional \\
\hline Masas sólido-quísticas & Teratoma maduro & Tumores primarios (la gran mayoría de ellos) \\
\hline \multirow{3}{*}{ Masas sólidas } & Miomas & Tumores primarios (menos frecuente) \\
& Fibromas & Metástasis \\
& Tecomas & \\
\hline
\end{tabular}

De forma general, las masas ováricas pueden dividirse en:

1. Masas quísticas que serán mayoritariamente benignas e incluyen lesiones quísticas uni o multiloculares, tanto neoplásicas como no neoplásicas (quistes funcionales, quistes de inclusión, quistes del paraovario, quistes paratubáricos, hidrosalpinx).

2. Masas sólido-quísticas, la gran mayoría malignas con algunas excepciones como el teratoma maduro.
3. Masas sólidas, encontrando en este apartado un amplio espectro que incluye desde benignas a malignas (miomas, fibromas, tecomas, T. Brenner, metástasis).

Para la caracterización de las masas ováricas se utilizan criterios morfológicos que son comunes a cualquier técnica de imagen y características de señal en resonancia magnética (RM) (Fig. 2). 


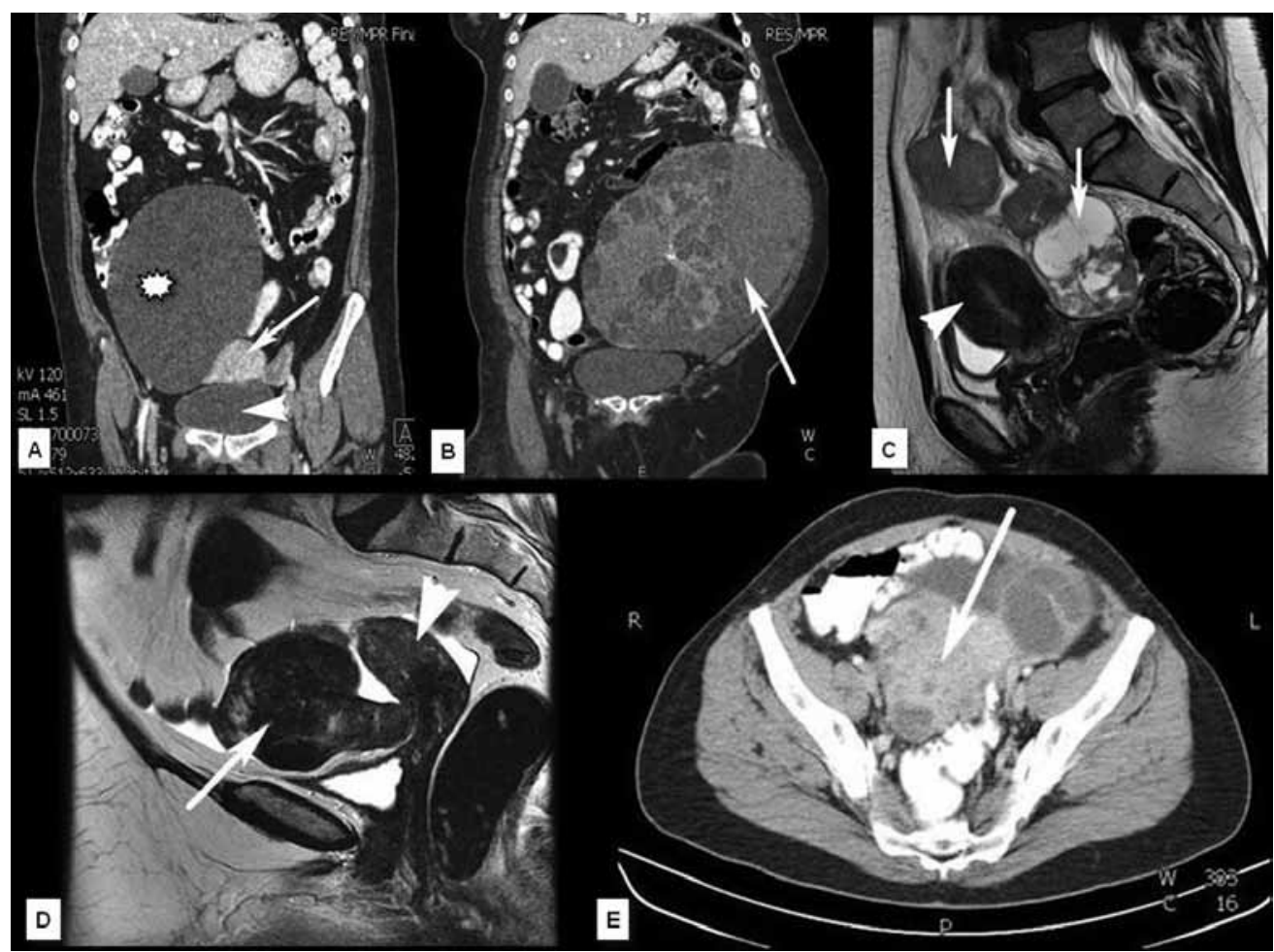

Figura 2. A: TC coronal. Lesión anexial quística benigna sin tabiques ni polos sólidos (estrella). Utero (flecha). Vejiga (cabeza de flecha). B: TC coronal. Masa anexial quística multiloculada con aspecto en vidriera. Tumor mucinoso benigno (flecha). C: RM sagital. Masa anexial sólido-quística maligna (flechas). Útero (cabeza de flecha). D: RM sagital. Masa anexial marcadamente hipointensa en T2. Fibroma ovárico (flecha). Útero (cabeza de flecha). E: TC axial. Masa anexial sólido-quística maligna (flecha).

- Criterios morfológicos de malignidad aplicables a cualquier técnica de imagen:

- Gran componente sólido

- Pared gruesa mayor de $3 \mathrm{~mm}$

- Tabiques o septos gruesos mayores de $3 \mathrm{~mm}$

- Proyecciones papilares o nódulos

- Áreas de necrosis

- Criterios auxiliaries:

- Ascitis

- Implantes peritoneales, mesentéricos u omentales

- Adenopatías

- Infiltración de pared u órganos pélvicos

Cuando se utilizan estos criterios la sensibilidad y especificidad para el diagnóstico de malignidad es de $91-92 \%$ y $91-$ $100 \%$, respectivamente ${ }^{2,6}$.
- Criterios de señal en RM

Proporcionan información que permite caracterizar ciertos tipos anatomopatológicos al identificar contenidos tales como grasa, sangre, tejido fibroso y colágeno, en cantidad abundante.

T1. Brillan sangre y grasa (con caída de señal en secuencias que la suprimen).

Permite la identificación de endometriomas y teratomas.

La metahemoglobina produce un acortamiento del T1 (hiperseñal). Las hemorragias cíclicas con alta viscosidad que contienen los quistes endometriósicos acortan el T2 (hiposeñal) y producen el llamado shading (sombreado).

Además de en los endometriomas podemos encontrar contenido hemático tam- 
bién en el hematosalpinx, quistes funcionales etc para cuyo diagnóstico diferencial con los endometriomas la RM arroja cifras inferiores en sensibilidad y especificidad. Los hematomas agudos que se producen en los quistes funcionales (isointensos en T1), contienen deoxihemoglobina que posteriormente (1 a 3 días), se oxida en su periferia pasando a metahemoglobina mostrando un anillo hiperintenso en $\mathrm{T} 1$.

Los quistes endometriósicos presentan hiperseñal en T1 e hiperseñal en T2 o el típico shading por las hemorragias repetidas.

La RM permite demostrar el contenido graso de los teratomas con alta intensidad de señal en T1 y con caída de señal en fase opuesta (que se basa en el artefacto de desplazamiento químico), en un $62-87 \%$ de los casos. Con técnicas específicas de supresión grasa se demuestra en un $92-95 \%{ }^{6}$.

T2. Hiposeñal muy acentuada de masas con alto contenido en tejido fibroso.

Así se identifican endometriomas, teratomas maduros o dermoides y tumores de alto contenido fibroso como leiomiomas, fibromas, fibrotecomas o tumor de Brenner (2-3\% de los tumores de ovario y raramente maligno, generalmente pequeño menor de $2 \mathrm{~cm}$, incidental y en un $30 \%$ asociado a otros tumores del ovario más frecuentemente tumores mucinosos) (Fig. 3).

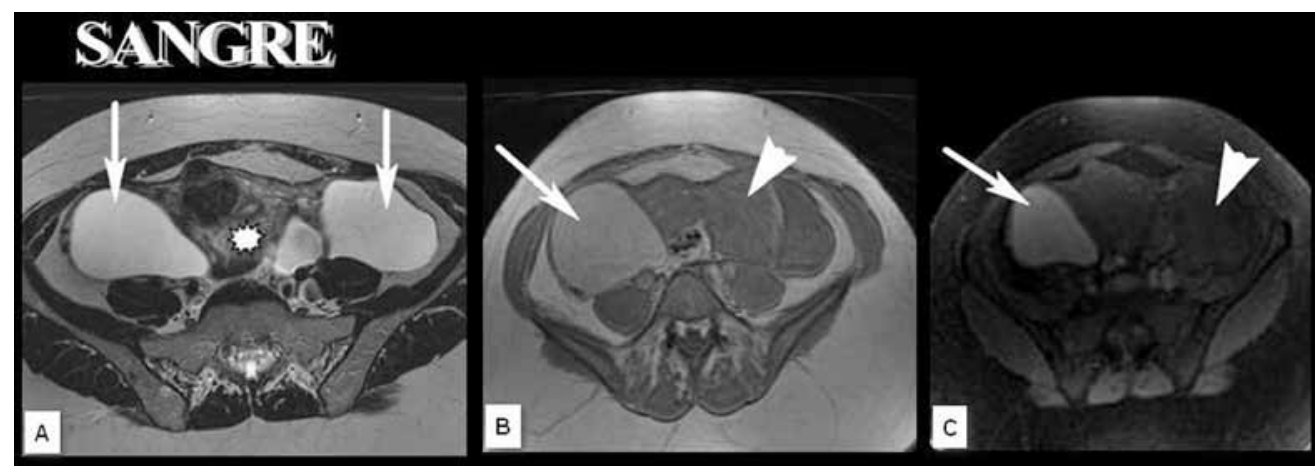

\section{GRASA}
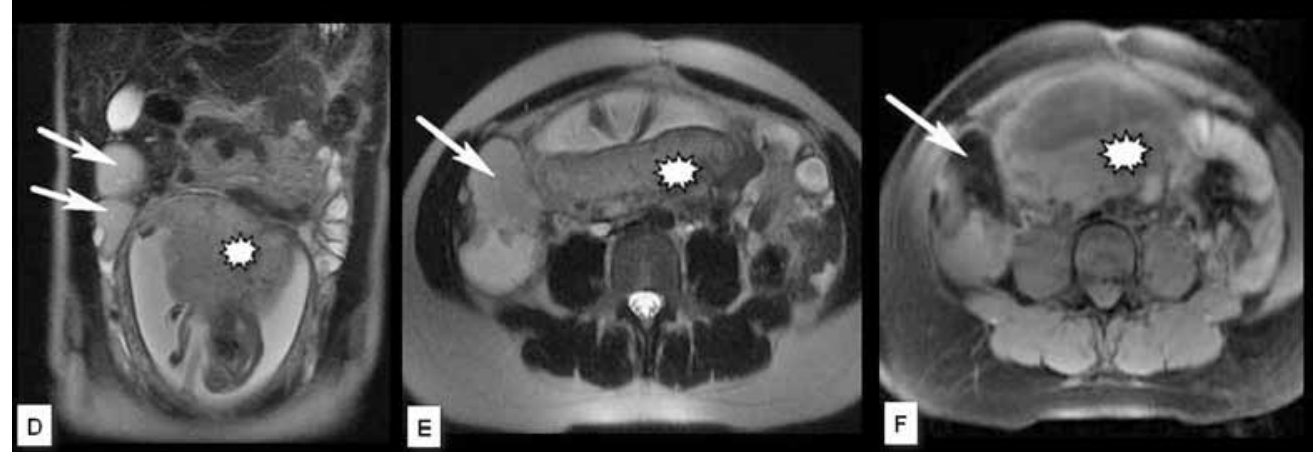

Figura 3. Caracterización tisular por RM. A: RM axial potenciada en T2. Masas quísticas anexiales bilaterales (flechas). Útero miomatoso (estrella). B: RM axial potenciada en T1. Hiperseñal en la masa del lado derecho (flecha) e hiposeñal en la del lado izquierdo (cabeza de flecha). C: RM axial potenciada en T1 con saturación grasa. La masa derecha mantiene su hiperseñal por lo que corresponde a contenido hemático (flecha). La del lado izquierdo mantiene su hiposeñal (cabeza de flecha). D: RM coronal potenciada en T2. Masa quística compleja en anejo derecho en paciente embarazada (flechas). Útero grávido (estrella). E: RM axial potenciada en T2. Masa quística compleja con polo sólido (flecha). Útero grávido (estrella). F: RM axial potenciada en T1 con saturación grasa. Caída de señal de gran parte de la lesión, lo que demuestra contenido graso (flecha) y es compatible con teratoma. Útero grávido (estrella). 
Respecto a algunas características especificas de tipos histológicos (permiten acortar el diagnóstico diferencial) mencionadas anteriormente, hay que tener en cuenta:

- Seroso versus mucinoso. Los tumores mucinosos suelen ser de mayor tamaño, multiloculados, con densidades o intensidades diferentes que le dan un aspecto en vidriera y son benignos en mayor proporción que los serosos. Los tumores serosos en un $30 \%$ presentan calcificaciones psammomatosas y con mayor frecuencia son bilaterales.

- Proyeccionas papilares. Altamente sugestivo de tumor epitelial y, si son muy profusas, de bajo potencial maligno (borderline) o malignos.

- Sincrónicos. En carcinomas endometrioides entre un $15-30 \%$, y otras veces asociados a hiperplasia endometrial (también puede verse esto en tumores de células de la granulosa, y ocasionalmente en tecomas y fibrotecomas).

- Presencia de grasa. Dato altamente específico de teratoma.

- Bilateralidad. Debe hacernos pensar en metástasis o carcinoma seroso.
- Tumores malignos en $2^{\mathrm{a}}$ y $3^{\mathrm{a}}$ década, hay que pensar en tumores de células germinales ${ }^{5,6}$.

\section{ESTADIFICACIÓN RADIOLÓGICA DEL CÁNCER DE OVARIO}

Clasificada por doble sistema, TNM (sistema de estadificación de la American Joint Committee on Cancer - AJCC) y FIGO (sistema de estadificación de la Federación Internacional de Ginecología y Obstetricia).

La estadificación del carcinoma de ovario es quirúrgica y patológica. Requiere una cirugía reglada que incluye histerectomía más doble anexectomía, y biopsias múltiples de los sitios más frecuentemente afectados, omento, repliegues peritoneales, cúpulas, ganglios pélvicos y paraaórticos (para estadificar con seguridad un IA todas las biopsias y ganglios serán negativos T1N0M0). Por otra parte, hallazgos relevantes clínicos o radiológicos antes de la cirugía pueden determinar la estadificación: adenopatías supraclaviculares palpables, metástasis pulmonares, pleurales o hepáticas, etc ${ }^{9}$.

Tabla 2. Estadificación de los tumores ováricos.

\begin{tabular}{|c|c|c|c|c|}
\hline Estadificación & & A & B & C \\
\hline Estadio I & $\begin{array}{l}\text { Tumor limitado al ova- } \\
\text { rio. } \\
\text { Sin células malignas en } \\
\text { ascitis o lavado perito- } \\
\text { neal. }\end{array}$ & $\begin{array}{l}\text { Limitado a un ovario. } \\
\text { Cápsula íntegra. } \\
\text { No tumor en la super- } \\
\text { ficie. }\end{array}$ & $\begin{array}{l}\text { Igual que A pero afecta- } \\
\text { ción bilateral. }\end{array}$ & $\begin{array}{l}\text { A o B más cápsula rota, } \\
\text { tumor en superficie del } \\
\text { ovario o positividad de } \\
\text { citología de líquido pe- } \\
\text { ritoneal. }\end{array}$ \\
\hline Estadio II & $\begin{array}{l}\text { Afectación uni o bila- } \\
\text { teral con extensión a } \\
\text { estructuras pélvicas. }\end{array}$ & $\begin{array}{l}\text { Extensión a útero y /o } \\
\text { trompas con citología } \\
\text { de líquido peritoneal } \\
\text { negativa. }\end{array}$ & $\begin{array}{l}\text { Extensión a otros teji- } \\
\text { do pélvicos (recto, ve- } \\
\text { jiga, paredes pélvicas). } \\
\text { Citología de líquido pe- } \\
\text { ritoneal negativa. }\end{array}$ & $\begin{array}{l}\text { A o B más positividad } \\
\text { de citología de líquido } \\
\text { peritoneal. }\end{array}$ \\
\hline Estadio III & $\begin{array}{l}\text { Tumor en cavidad } \\
\text { peritoneal fuera de la } \\
\text { pelvis o ganglios regio- } \\
\text { nales positivos. }\end{array}$ & $\begin{array}{l}\text { Metástasis peritonea- } \\
\text { les microscópicas fue- } \\
\text { ra de la pelvis. }\end{array}$ & $\begin{array}{l}\text { Metástasis peritonea- } \\
\text { les fuera de la pelvis } \\
\leq 2 \mathrm{~cm} \text {. }\end{array}$ & $\begin{array}{l}\text { Metástasis peritonea- } \\
\text { les fuera de la pelvis } \\
>2 \mathrm{~cm} \text { y/o metástasis } \\
\text { ganglionares regiona- } \\
\text { les. }\end{array}$ \\
\hline Estadio IV & $\begin{array}{l}\text { Metástasis a distancia } \\
\text { excluidas las perito- } \\
\text { neales. }\end{array}$ & & & \\
\hline
\end{tabular}


La cirugía óptima es un factor determinante en el control de la enfermedad y en la supervivencia. La estadificación proporciona información para la toma de decisiones e instauración del tratamiento adecuado, así el rol de la TC preoperatoria es identificar y seleccionar' ${ }^{2}$ :

- Pacientes candidatas a cirugía óptima, proporcionando información sobre la enfermedad localizada en áreas más difícilmente abordables en la cirugía (hilio esplénico, cúpulas diafragmáticas, etc.).

- Pacientes candidatas a neoadyuvancia por presentar criterios de irresecabilidad (implantes en la raíz del mesenterio, afectación de la porta hepatis, infiltración de paredes pélvicas, adenopatías por encima de los hilios renales, implantes en saco menor, hidronefrosis...) o que requieren la participación de otros equipos quirúrgi$\cos$ (resecciones intestinales etc).

- Pacientes con carcinomatosis de otro origen, por las implicaciones pronósticas y terapéuticas, como se ha mencionado con anterioridad.

Las vías de diseminación principales en el carcinoma de ovario serán invasión directa y diseminación peritoneal. La enfermedad limitada a la pelvis incluye los estadios precoces I y II.

Estadio I. Tumor limitado al ovario (Fig. 4) IA y IB - uno o ambos ovarios con cápsula íntegra y IC rotura capsular- tumor en la superficie o líquido positivo.

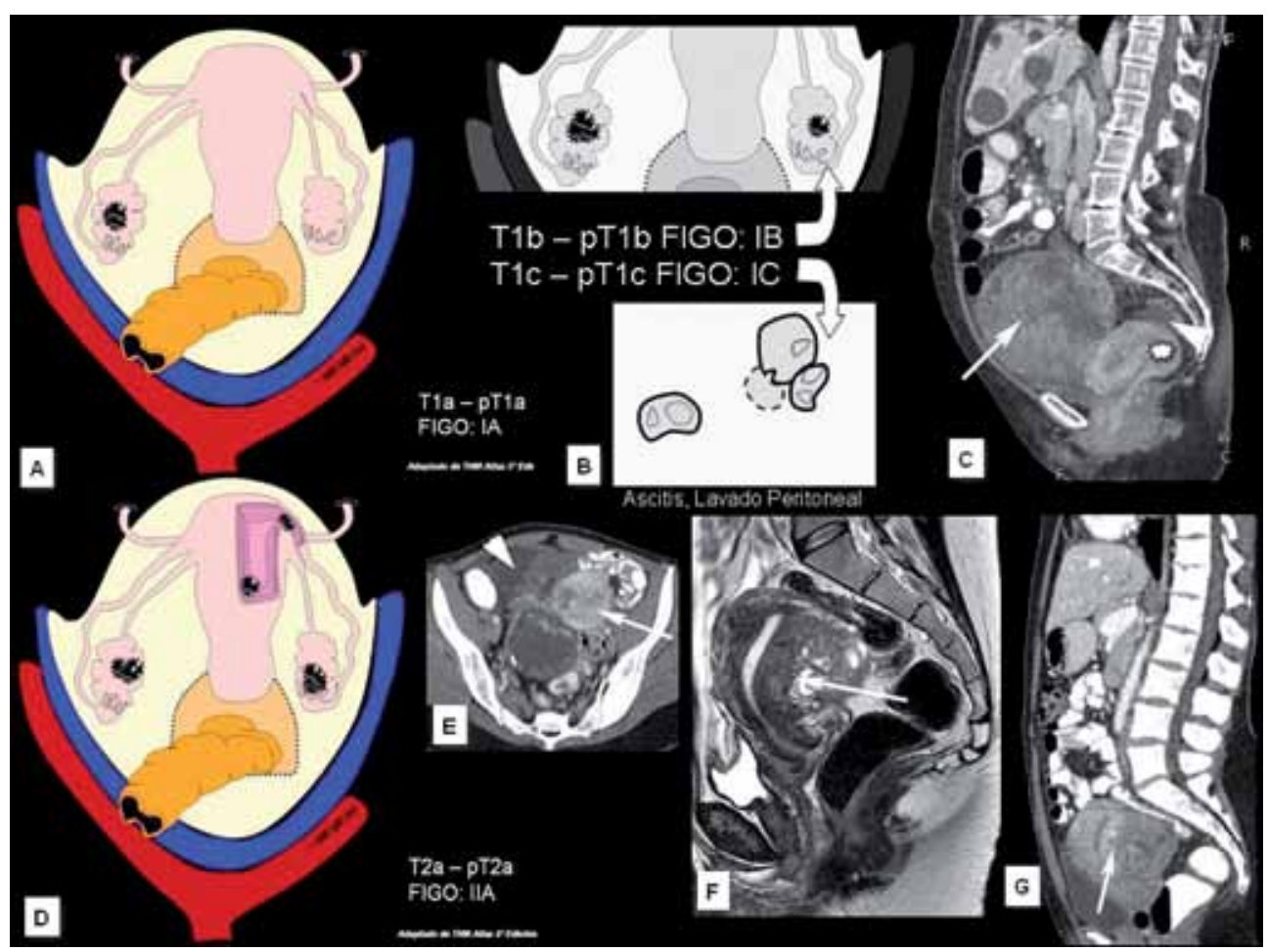

Figura 4. Estadificación. A: Esquema FIGO IA. B: Esquema FIGO IB (afectación ovárica bilateral) y FIGO IC (con líquido ascítico postivo para células tumorales). C: TC sagital. Masa anexial derecha (flecha) con líquido ascítico (cabeza de flecha), sin células tumorales, estadio IA. Útero (estrella). D: Esquema FIGO IIA. E: TC axial. Masa anexial derecha (cabeza de flecha) con infiltración uterina (flecha). F: RM sagital potenciada en T2 (otra paciente). Masa anexial con infiltración de pared posterior de útero (FIGO IIA) (flecha). G: TC multicorte reconstrucción sagital de la misma paciente (flecha). 
Estadio II. Afectación de uno o dos ovarios con extensión a otras estructuras pélvicas:

- IIA: extensión a útero y trompas (líquido negativo).

- IIB: extensión a otros tejidos pélvicos (recto, vejiga, paredes pélvicas) (líquido negativo).
- II C: criterios del A o el B con líquido positivo.

La presencia de líquido ascítico sin células tumorales no supone un estadio III.

Los estadios avanzados III y IV incluyen la diseminación peritoneal fuera de la pelvis y las metástasis a distancia (Fig. 5).

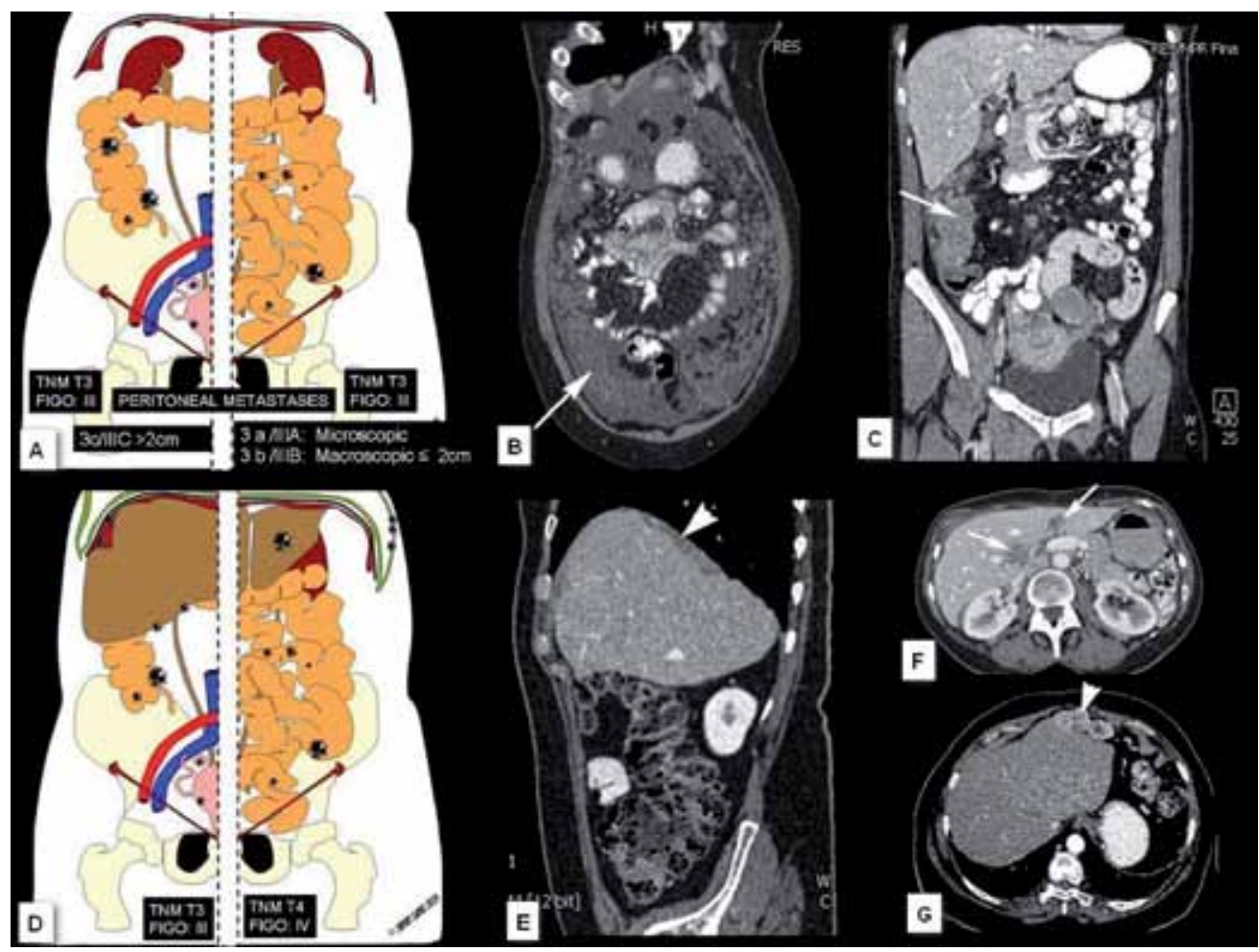

Figura 5. Estadificación. A: Esquema FIGO III. B: Múltiples implantes omentales que configuran el llamado omental cake (flecha). C: Implante de gran tamaño en la pared del colon derecho (flecha). D: Esquema FIGO III (implante superficie hepática) y FIGO IV. E: Implantes subdiafragmáticos y en cúpula hepática (cabeza de flecha). F: Implantes intercisurales superficiales hepáticos, no intraparenquimatosos FIGO III (flechas). G: Implantes en superficie con invasión parenquimatosa (cabeza de flecha).

El $70 \%$ de las pacientes son diagnosticadas en estadio III y tienen afectación peritoneal al diagnóstico. Los sitios mas comúnmente afectados son el omento, la región subfrénica dcha y el fondo de saco de Douglas. Esto es debido a las rutas de circulación del líquido peritoneal, desde las partes más declives en el fondo de saco de Douglas a las zonas más craneales siendo reabsorbido, a través de una rica red de linfáticos del diafragma, hasta nódulos linfáticos mediastínicos anteriores. Estos linfáticos diafragmáticos pueden estar obstruidos por células tumorales lo que 
bloquea la absorción del líquido y contribuye a la ascitis maligna. La circulación del líquido está determinada por múltiples gradientes de presión en los que influyen varios factores (como la respiración, movimientos peristálticos, la gravedad) y características anatómicas como el anclaje de la raíz del mesenterio y el ligamento freno cólico izquierdo, etc.).
Estadio III. Metástasis peritoneales fuera de la pelvis:

- III A microscópicas

- III B macroscópicas menores de $2 \mathrm{~cm}$

- III C mayor de $2 \mathrm{~cm}$ y/o adenopatías regionales

Estadio IV. Metástasis a distancia (excluidos los implantes peritoneales) (Fig. 6).

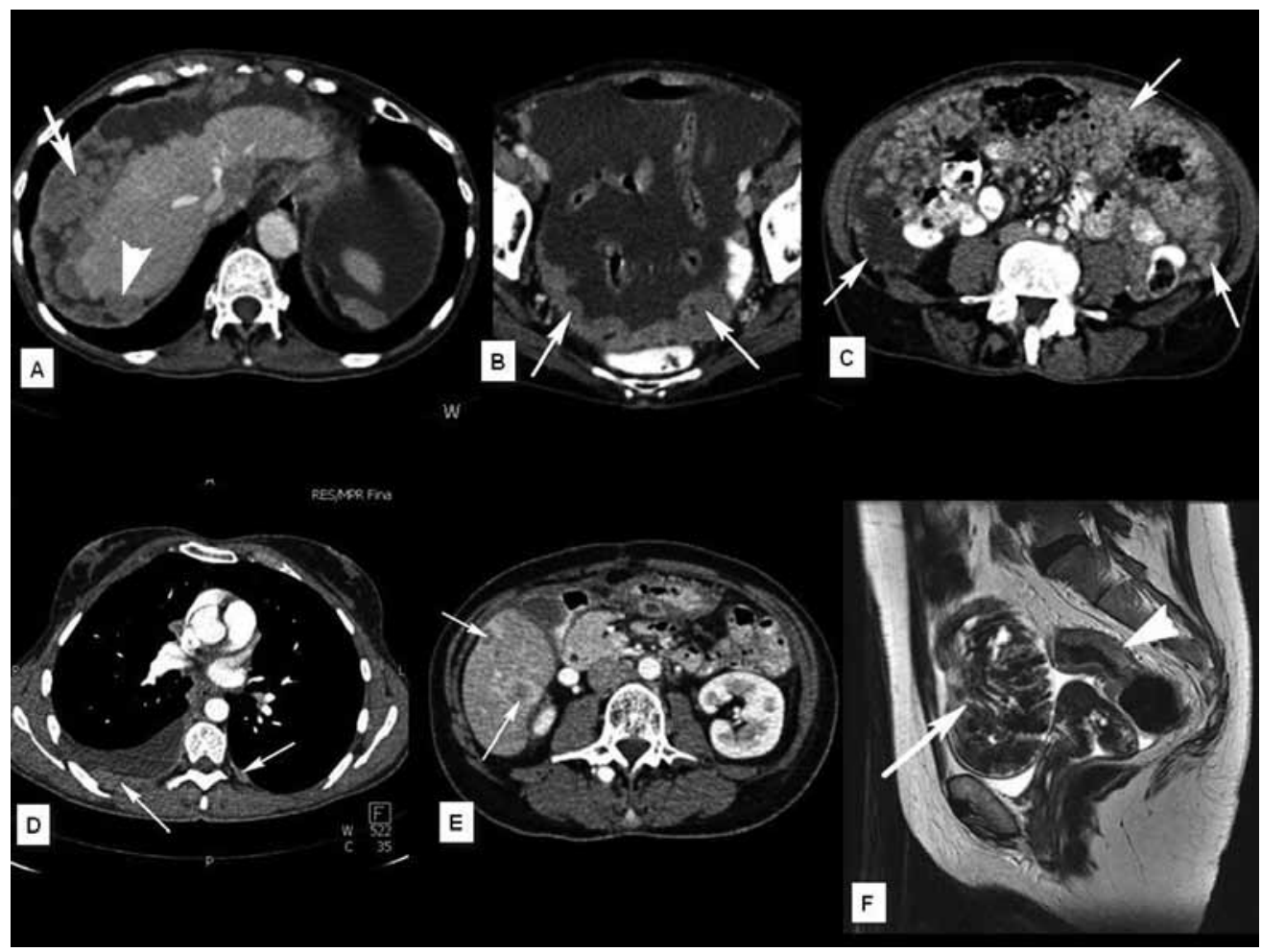

Figura 6. Enfermedad avanzada. A: Múltiples implantes en cúpula (flecha) y superficie hepática (cabeza de flecha). B: Implantes en peritoneo parietal posterior (flechas). C: Múltiples implantes peritoneales y mesentéricos (flechas). D: Metástasis pleurales (flechas). E: Metástasis hepáticas (flechas). F: Metástasis en ovario (flecha) de carcinoma de recto (cabeza de flecha).

La TCMC ha contribuído a un mejor diagnóstico de lesiones subcentimétricas y de implantes en localizaciones difíciles tales como las cúpulas diafragmáticas, y la superficie hepática, incluyendo las intercisurales, siendo especialmente relevante el diagnóstico diferencial entre implantes invasivos $(9 \%)$ y no invasivos $(91 \%)^{10,11}$.
Las metástasis a través del drenaje linfático se producen por tres vías. La red principal sigue la vena ovárica hacia ganglios para aórticos y paracava hasta la altura de los hilios renales, siendo éste el sitio más frecuente de adenopatías. Una segunda red lo hace a través del ligamento ancho hacia ganglios pélvicos incluyendo la ca- 
dena ilíaca externa e interna y una tercera red a través del ligamento redondo hacia ganglios inguinales.

Las metástasis hematógenas son la vía menos común, y no suele encontrarse en el momento del diagnóstico, pero se ven en enfermedad recurrente y se han demostrado en más del $50 \%$ de las pacientes en series de autopsias (hígado, pulmón, cerebro, hueso, suprarrenales, riñones y bazo) ${ }^{12}$.

\section{VALORACIÓN DE RESPUESTA, EVOLUCIÓN Y COMPLICACIONES}

La técnica de imagen indicada para valoración de respuesta, control evolutivo de posibles recaídas y complicaciones será la TC. (Comentario: al final de la página se recoge la controversia en función de los diferentes autores con respecto a la mejor técnica en el seguimiento y detección de recidiva de enfermedad).

La indicación de la PET/TC permanece controvertida según autores ${ }^{2,4,12}$.

En la detección primaria de carcinoma de ovario la PET ha demostrado sensibilidad del 52 al 58\% y especificidad del 76 al $78 \%$ por lo que no está recomendada para el diagnóstico inicial. Existen falsos negativos con tumores precoces, de bajo grado o borderlines y falsos-positivos en hidrosalpinx, fibromas pediculados, endometriosis. En mujeres premenopáusicas sometidas a controles por otras neoplasias se han interpretado como metástasis u ovarios hipermetabólicos cuando en realidad se trataba de cuerpos lúteos precoces o folículos tardíos. Por el contrario un ovario hipermetabólico en una mujer postmenopáusica debe ser considerado potencialmente como maligno (la captación ovárica en la PET debe interpretarse teniendo en cuenta el momento del ciclo menstrual de la mujer).

Aunque no es la técnica de elección para la detección se está expandiendo para planificación de tratamiento o seguimiento. La PET/TC con contraste mejora la precisión de la estadificación y se recomienda cada vez más para el diagnóstico de recaí- das. En un meta-análisis publicado en el Eur J Radiol de 2009 comparando las diferentes técnicas, se demuestran los siguientes resultados ${ }^{13}$ :

- PET/TC sensibilidad 91\% y especificidad $88 \%$

- TC sensibilidad del 79\% y especificidad $84 \%$

- RM sensibilidad del 75\% y especificidad $78 \%$

Y en AJR 2010 concluyen que la PET/CT es la mejor técnica para detectar lesiones en el seguimiento y sospecha de recaída en mujeres con carcinoma de ovario ${ }^{2}$. Sin embargo en un estudio preliminar de Radiology en octubre de $2010^{14}$ sus datos sugieren precisión similar entre la TC con contraste y la PET/TC en el diagnóstico de recurrencias. Además, preconizan que el tamaño, número y SUV (del inglés standard uptake value-valor estandarizado de captación) máximo de la recurrencia tienen potencial como biomarcadores pronósticos

\section{TÉCNICAS EMERGENTES}

Las técnicas de imagen utilizadas, hasta ahora, en el estudio de los tumores han sido fundamentalmente morfológicas y han conseguido una alta resolución espacial, gran velocidad de adquisición y junto con los medios de contraste alta resolución anatómica y de contraste. El mejor conocimiento de la biología tumoral y los avances en biología celular, molecular y en genética, nos han permitido conocer mejor las bases biológicas del cáncer y una serie de procesos que tienen lugar en los tumores y que van cobrando especial relevancia como son hipoxia, celularidad, angiogénesis etc. y que no pueden ser valoradas por las técnicas convencionales.

Además, el gran avance terapéutico de la radioterapia con protocolos que permiten definir subvolúmenes guiados por la imagen y la introducción de nuevos fármacos diana en oncología médica requieren de la imagen una nueva valoración de los tumores y nuevos parámetros para evaluación de respuesta al tratamiento, incluso la 
selección precoz de pacientes que responderán o no a nuevas terapias.

Todo ello ha permitido el desarrollo de nuevas técnicas conocidas como imagen funcional que es capaz de estudiar in vivo procesos fisiológicos de los tejidos y de los tumores ${ }^{15,16}$. Así realizaremos estudios de perfusión que valoran la angiogénesis, estudios del metabolismo tumoral con técnicas de PET o espectroscopia por RM, de celularidad mediante difusión (DWI) por RM, y obtendremos información sobre niveles de oxigenación, proliferación celular o vascularización tumoral.

La aplicación actual (no suficientemente contrastada) de estas técnicas en el cáncer de ovario es fundamentalmente en dos tipos de exploraciones: estudios dinámicos con contraste en RM, que se correlacionan con la angiogénesis tumoral buscando patrones de realce que permitan distinguir tumores epiteliales de ovario benignos, borderline y tumores invasivos ${ }^{17}$, y estudios de Difusión (DWI) en diseminación peritoneal de cáncer de ovario que parece pueden mejorar la detección y delimitación de los implantes peritoneales tanto en el diagnóstico inicial como en el seguimiento, y diferenciar cambios en el tumor inducidos por el tratamiento. La cuantificación de sus valores parece resultar útil en la monitorización de la respuesta al tratamiento. No obstante, la interpretación de las imágenes en difusión deben ser siempre correlacionadas con la imagen convencional de RM para obtener el máximo rendimiento de éstas ${ }^{18}$.

\section{CONSIDERACIONES FINALES}

La aportación de los estudios de imagen en el manejo del cáncer de ovario será:

- RM en la caracterización de lesiones indeterminadas.

- TCMC para optimizar el procedimiento quirúrgico y la estrategia terapéutica.

Las técnicas emergentes como la PET/ TC, estudios dinámicos, perfusión y difusión de forma preliminar demuestran una mejora en la precisión diagnóstica tanto en la caracterización como en la estadificación y en el control evolutivo para el diagnóstico de recaídas y valoración de respuesta al tratamiento.

\section{BIBLIOGRAFÍA}

1. SAKsouk FA, Johnson SC. Recognition of the ovaries and ovarian origin of pelvic masses with CT. Radiographics 2004; 24: S133-S146.

2. LYER VR, LEE SI. CRI, CT, and PET/CT for ovarian cancer detection and adnexal lesion characterization. Am J Roentgenol 2010; 194: 311-321.

3. Pickhardt PJ, Hanson ME. Incidental adnexal masses detected at low-dose unenhanced $\mathrm{CT}$ in asymtomatic women age 50 and older: Implications for clinical management and ovarian cancer screening. Radiology 2010; 257: 144-150.

4. BaLACHANDRAN A, IYeR RB. Imaging of ovarian cancer. Appl Radiol 2005; 34: 19-29.

5. Jung SE, LeE JM, Rha SE, Byun JY, Jung JI, Hahn ST. CT and MR imaging of ovarian tumors with emphasis on differential diagnosis. Radiographics 2002; 22: 1305-1325.

6. IMAoka I, Wada A, KaJI Y, Hayashi T, Hayashi M, Matsuo $M$ et al. Developing an MR imaging strategy for diagnosis of ovarian masses. Radiograhics 2006; 26: 1431-1448.

7. Takeuchi M, Matsuzaki K, Uehara $H$, Nishitani $H$. Malignant transformation of pelvic endometriosis: MR imaging finding and pathologic correlation. Radiographics 2006; 26: 407-417.

8. Kinkel K, Lu Y, Mehdizade A, Pelte MF, HricaK $\mathrm{H}$. Indeterminate ovarian mass at US: incremental value of second imaging test for Characterization - Meta-analysis and bayesian analysis. Radiology 2005; 236: 85-94.

9. American Joint Committee on Cancer (AJCC). Cancer staging manual. 7th Edition. Springer, 2010.

10. Pannu HK, Bristow RE, Montz FJ, Fishman EK. Multidetector CT of peritoneal carcinomatosis from ovarian cancer. Radiographics 2003; 23: 687-701.

11. AKIN O, Sala E, Moskowits CS, Ishill N, Soslow RA, CHI DS et al. Perihepatic metastasis form ovarian cancer: sensitiviy and specificity of CT for detection of metastasis with and those without liver parenchymal invasion. Radiology 2008; 248: 511-517. 
12. Woodward PJ, Hosseinzadeh K, SaEnger JS. From the archives of the AFIP. Radiologic staging of ovarian carcinoma with patologic correlation. Radiographics 2004; 24: 225246.

13. Gu P, Pan LL, Wu SQ, Sun L, Huang G. CA 125, PET alone, PET-CT and MRI in diagnosing recurrent ovarian carcinoma: a systematic review and meta-analysis. Eur J Radiol 2009; 71: 164-174.

14. Sala E. Kataoka M, Pndit-taskar N, Ishill N, MiRONOv S, Moskowitz CS et al. Recurrent ovarian cancer: use of contrast-enhanced CT and PET/CT to accurately localize tumor recurrence and to predit patients' survival. Radiology 2010; 257: 125-134.
15. García Figueiras R, Padhani AR, Vilanova JC, Goh V, Villalba Martin C. Imagen funcional tumoral. Parte 1. Radiología 2010; 52: 115-125.

16. García Figueiras R, Padhani AR, Vilanova JC, GoH $\mathrm{V}$, Villalba Martin C. Imagen funcional tumoral. Parte 2. Radiología 2010; 52: 208-220.

17. Thomassin-Naggara I, Bazot M, Daraï E, Callard P, Thomassin J, Cuenod CA. Epitelial ovarian tumors: value of dynamic contrast-enhanced MR imaging and correlation with tumor angiogenesis. Radiology 2008; 248: 148-159.

18. Kyriazi S, Colins DJ, Morgan VA, Giles SL, DE SouzA NM. Diffusion-weighted imaging of peritoneal disease for noninvasive staging of advanced ovarian cancer. Radiographics 2010; 30: 1269-1285. 